\title{
Invertebrados exóticos nuevos o poco conocidos (Tricladida, Gastropoda, Diplopoda, Symphyla, Isopoda, Arachnida) en la Ciudad de Buenos Aires, Argentina
}

New or Little Known Exotic Invertebrates (Tricladida, Gastropoda, Diplopoda, Symphyla, Isopoda, Arachnida) in Buenos Aires City, Argentina

Federico Agnolin ${ }^{1,2^{*}}$, Agustín Agnolin ${ }^{3}$, Jordi Garcia-Marsà2 ${ }^{2}$ Nicolás Olejnik ${ }^{3}$

1 Laboratorio de Anatomía Comparada y Evolución de los Vertebrados, Museo Argentino de Ciencias Naturales "Bernardino Rivadavia". Av. Ángel Gallardo 470, (C1405DJR) Buenos Aires, Argentina.fedeagnolin@yahoo.com.ar

2 Fundación de Historia Natural "Félix de Azara". Universidad Maimónides, Hidalgo 775, (C1405BDB) Buenos Aires, República Argentina.

3 Instituto Nacional de Antropología y Pensamiento Latinoamericano, 3 de Febrero 1378, (C1426BJN) Buenos Aires, Argentina. agusagnolin@yahoo.com.ar

\section{RESUMEN}

La fauna de planarias terrestres (Tricladida, Geoplanoidea), babosas (Mollusca, Gastropoda), diplópodos (Myriapoda, Diplopoda), sínfilos (Myriapoda, Symphylla) y arácnidos (Chelicerata) de la Ciudad Autónoma de Buenos Aires (CABA, Argentina) permanece pobremente conocida. En la presente contribución se dan a conocer nuevos registros de especies de estos grupos, naturalizadas en el ámbito urbano de CABA y alrededores. Las colectas y observaciones fueron realizadas sin un método sistemático. Se reportan: Rhynchodemus sylvaticus (Tricladida, Geoplanidas; primer registro para la provincia de Buenos Aires y segundo para Argentina), Obama nungara (Tricladida, Geoplanidae; primera cita para CABA), Imbira flavonigra (Tricladida, Geoplanidae; primer registro para Argentina), Milax gagates (Mollusca, Milacidae; primer registro para CABA), Cylindroiulus latestriatus (Diplopoda, Julidae; primer registro para Argentina), Ophyiulus pilosus (Diplopoda, Julidae; primer registro para

\footnotetext{
- Ref. bibliográfica: Agnolin F.; Agnolin, A.; Garcia-Marsà, J.; Olejnik, N. 2020. "Invertebrados exóticos nuevos o poco conocidos (Tricladida, Gastropoda, Diplopoda, Symphyla, Isopoda, Arachnida) en la Ciudad de buenois Aires, Argentina". Acta zoológica lilloana 64 (1): 13-29. Fundación Miguel Lillo, Tucumán, Argentina. D.O.I.: https://doi.org/10.30550/j.azl/2020.64.1/2

> Recibido: 2 de enero de 2020 - Aceptado: 6 de abril de 2020 
Argentina), Brachydesmus superus (Diplopoda, Paradoxomatidae; primer registro para CABA y segundo para Argentina), Symphylella vulgaris (Symphyla, Scolopendrellidae; primer registro para Argentina), Cylisticus convexus (Oniscidea, Cylisticidae; segunda cita para Argentina, luego de 90 años), Uloborus plumipes (Arachnida, Uloboridae; segunda cita para CABA y para Argentina) y Leucauge argyra (Arachnida, Tetragnathidae; primera cita para la provincia de Buenos Aires y segunda cita para Argentina). Diversos autores indican para Europa un rápido incremento en el número de especies invertebradas introducidas, un patrón extrapolable a Argentina. Lamentablemente, hasta la fecha, no se cuenta con datos certeros acerca de los patrones de distribución o abundancia de estas especies. En este sentido es necesario priorizar su conocimiento, incluso de aquellas potencialmente perjudiciales para ambientes nativos.

Palabras clave - Ciudad Autónoma de Buenos Aires, invertebrados exóticos, nuevos registros.

\section{ABSTRACT}

The terrestrial flatworm (Tricladida, Geoplanoidea), slug (Mollusca, Gastropoda), diplopodan (Myriapoda, Diplopoda), symphilan (Myriapoda, Symphylla) and spider (Chelicerata) faunas of Buenos Aires City (CABA, Argentina) are poorly known. The aim of the present contribution is to report new records of exotic species belonging to those clades. Collection and observation of specimens were made without a systematic method. The following species are reported: Rhynchodemus sylvaticus (Tricladida, Geoplanidae; first record for Buenos Aires province and second record for tArgentina), Obama nungara (Tricladida, Geoplanidae; CABA), Imbira flavonigra (Tricladida, Geoplanidae; first record for Argentina), Milax gagates (Mollusca, Milacidae; first record for CABA), Cylindroiulus latestriatus (Diplopoda, Julidae; first record for Argentina), Ophyiulus pilosus (Diplopoda, Julidae; first record for Argentina), Brachydesmus superus (Diplopoda, Paradoxomatidae; first record for CABA and second record for tArgentina), Symphylella vulgaris (Symphyla, Scolopendrellidae; first record for Argentina), Cylisticus convexus (Oniscidea, Cylisticidae; second record for the city by most than 90 years), Uloborus plumipes (Arachnida, Uloboridae; second record for CABA and the country), and Leucauge argyra (Arachnida, Tetragnathidae; first record for Buenos Aires province and second record for Argentina). Several authors have indicated that the number of exotic invertebrates in European cities has been dramatically increased in the last years. This pattern should be extrapolated to Argentina. Unfortunately, up to the date we entirely lack information about dispersal and distribution of exotic invertebrates in the city. It is necessary to prioritize the study of exotic species, especially those that are potentially problematic for native environments.

Keywords - Buenos Aires City, exotic invertebrates, new records. 


\section{INTRODUCCIÓN}

La Ciudad Autónoma de Buenos Aires constituye el tejido urbano de mayor tamaño en Argentina. A pesar de su importancia y accesibilidad, la flora y fauna locales aún distan de ser bien conocidas. Publicaciones relacionadas a su fauna local son escasas, y poco sabemos acerca de las especies de invertebrados que puede encontrarse en plazas y jardines urbanos (Agnolin et al., 2019).

Varios autores (Hänggi y Straub, 2016) han resaltado que en zonas metropolitanas, las especies invasoras se pueden dispersar, establecer poblaciones estables y transformarse potencialmente en perjudiciales para la fauna local. Sin embargo, aunque desconocemos aún (especialmente en lo que a Argentina atañe) el impacto que estas nuevas especies pueden ejercer sobre las especies nativas, es necesario saber al menos que especies exóticas están presentes en el área.

Las publicaciones que tratan sobre invertebrados sinantrópicos argentinos son escasas, en consecuencia, hay una gran deficiencia de datos al respecto. Debido a la escasez de información publicada, no es posible conocer el comportamiento y la distribución geográfica de estas especies exóticas.

De esta manera, la publicación de nuevos registros y todo tipo de información relacionada a la distribución de especies exóticas (Hänggi y Straub, 2016) es de especial importancia. Sólo sobre esta base se podrán reconocer patrones de expansión e invasión de especies foráneas.

El objetivo de esta contribución es brindar información novedosa de especies no autóctonas o poco conocidas de invertebrados en la Ciudad Autónoma de Buenos Aires y alrededores.

\section{MATERIALES Y MÉTODOS}

Se recolectaron especímenes de Tricladida, Mollusca y Myriapoda hallados en dos jardines de la Ciudad Autónoma de Buenos Aires y se determinaron sobre la base de características morfológicas externas mediante la bibliografía específica para cada grupo. En el caso de Milax gagates, mediante la utilización de lupa estereoscópica, se practicó una disección del ejemplar con la finalidad de reconocer su anatomía interna.

El material de referencia proviene del patio de una vivienda privada de una zona densamente poblada en plena ciudad de Buenos Aires (barrio de Liniers) que cuenta con plantas decorativas tanto nativas como exóticas, con piedras y ladrillos que brindan refugio a los invertebrados. Se trata de un jardín interno que carece de conexión directa con otras áreas verdes o jardines. Su extensión aproximada es de unos 6 metros cuadrados.

La colecta y observaciones fueron realizadas sin un método prestablecido, mediante la búsqueda activa, ya sea a ojo desnudo o volteando troncos, piedras y macetas. La cantidad de especímenes colectados dependió de su abundancia local.

Adicionalmente, se recolectaron especímenes de algunas especies en los Parques Centenario y Avellaneda, ubicados en los barrios de Caballito y Parque Avellaneda, 
respectivamente, en la ciudad de Buenos Aires. En estos casos los materiales se obtuvieron en un ambiente abierto con arboledas de plantas exóticas, ladrillos y troncos que sirven de refugio a estos invertebrados.

Los ejemplares obtenidos fueron fijados en formol al 10\%, conservados en alcohol 70 \%, y depositados en la Colección de Invertebrados (CFA-In) y Colección de Artrópodos (CFA-Ar) de la Fundación de Historia Natural "Félix de Azara", Ciudad Autónoma de Buenos Aires, Argentina.

\section{RESULTADOS}

Tricladida Lang, 1884

Geoplanidae Stimpson, 1857

Rhynchodemus sylvaticus (Leidy, 1851)

Material referido.- CFA-In-5601, ejemplar adulto recolectado. El especímen fue hallado debajo de ladrillos en un jardín interno en el barrio de Liniers, Ciudad Autónoma de Buenos Aires, Argentina.

Comentarios.- El ejemplar es referible a $R$. sylvaticus por presentar la siguiente combinación única de caracteres: cuerpo de sección subcircular, el extremo cefálico de color negruzco, en extremo anterior con dos ojos negros, una mancha oscura transversal en faringe y dorso color pardo oscuro, recorrido por dos franjas negras longitudinales sub-paralelas (Negrete, 2013; Álvarez Presas et al., 2014).

$R$. sylvaticus sería una especie oriunda de Europa, posiblemente introducida en el continente americano (Jones, 1998), y debería confirmarse su distribución en el pasado. Ha sido registrada en Estados Unidos, donde frecuenta bosques cercanos a grandes ciudades y ambientes urbanos como invernaderos y jardines internos $(\mathrm{Hy}-$ man, 1943; Vila-Farré et al., 2011; Negrete, 2013). En Argentina cuenta con registros previos en la ciudad de San Antonio, provincia de Misiones, donde los ejemplares fueron hallados en jardines (debajo de macetas y ladrillos) (Negrete, 2013).

El presente ejemplar constituye la segunda cita para la especie en Argentina, y la primera en la provincia de Buenos Aires, lo que representa una expansión de aproximadamente 1.200 kilómetros con respecto a la localidad citada por Negrete (2013).

\section{Imbira flavonigra Amaral, Ribeiro, Müller, Valiati \\ y Leal-Zanchhet, 2018}

Material referido. - CFA-In-5602, 4 ejemplares recolectados. Los especímenes fueron hallados debajo de un tronco en una arboleda artificial emplazada en el Jardín Botánico "Carlos Thays", ubicado en el Barrio de Palermo, Ciudad Autónoma de Buenos Aires, Argentina (Figura 1A). 
Comentarios. - Se trata de una especie muy llamativa, difícilmente confundible con otro taxón. Imbira flavonigra se caracteriza externamente por presentar la superficie dorsal con una banda longitudinal amarilla bordeada por dos bandas negras que contrastan con el resto del cuerpo de color grisáceo (Amaral et al., 2018).

Esta especie era conocida hasta el día de la fecha solo para su localidad típica Maquiné, en el estado de Río Grande do Sul, Brazil. Los ejemplares sobre los que se basó proceden de un área muy restringida de selva lluviosa emplazada en la región biogeográfica conocida como "Mata Atlántica” (Amaral et al., 2018). Amaral et al. (2018) propusieron, debido a sus requerimientos ecológicos especiales y a su restringida distribución geográfica, que Imbira flavonigra sería un endemismo local. El hallazgo de esta especie en un bosque artificial emplazado en una gran urbe, donde era notablemente abundante (observamos decenas de individuos), sugiere que los requerimientos ecológicos serían más versátiles. De hecho, sobre la base de la abundancia de los ejemplares observados, es posible que sea una especie invasora exitosa.

El presente hallazgo constituye el primero para la especie en Argentina y el único por fuera de la localidad tipo.

\section{Obama nungara Carbayo, Álvarez-Presas, Jones y Riutort, 2016}

Material referido. - CFA-In-5603, 5 ejemplares recolectados, conservados en alcohol. Los especímenes fueron hallados debajo de troncos en una arboleda exótica de Álamo blanco (Populus alba) en el Parque Centenario, barrio de Caballito, Ciudad Autónoma de Buenos Aires, Argentina. Los ejemplares fotografiados han sido observados e identificados en el lugar, pero no recolectados, en la localidad de Ramos Mejía, Partido de La Matanza, Provincia de Buenos Aires, Argentina y en La Reja, Partido de Moreno, Provincia de Buenos Aires, Argentina (Figura 1B-D). Fueron hallados bajo troncos y grandes rocas.

Comentarios. - O. nungara es fácilmente distinguible de otras especies cercanas por el dorso de color amarillento con punteado marrón oscuro a negro, lo que da una apariencia casi negra al animal en vida (ocasionalmente los ejemplares pueden exhibir una banda media longitudinal amarillenta). Los ojos se distribuyen hasta alcanzar 1/3 del ancho del cuerpo, y la apertura bucal está ubicada anteriormente (Carbayo et al., 2016). La especie es nativa de los estados de Santa Catarina y Río grande do Sul, en Brazil, y el noreste de Argentina (Lago Barcia et al., 2019). Hoy en día es invasora en Europa, incluyendo Inglaterra, España, Francia Bélgica e Italia (Soors et al., 2019; Justine et al., 2020). En Argentina ha sido previamente citado bajo el nombre Obama marmorata para las localidades bonaerenses de La Plata, Los Toldos y Nueve de Julio (Lago Barcia et al., 2015). En correspondencia con lo observado en la presente contribución, Lago Barcia et al. $(2015,2019)$ indican que los ejemplares por ellos citados se encontraron en varios jardines y viveros de plantas, ocultos debajo de macetas y otros objetos que se encuentran en el suelo, donde po- 
dían ser abundantes. Carbayo et al. (2016) la consideran una especie peridoméstica, exclusiva de ambientes disturbados.

Mollusca Linnaeus, 1758

Gastropoda Cuvier, 1797

Milacidae Ellis, 1926

Milax gagates (Draparnaud, 1801)

Material referido. - CFA-In-5558, ejemplar adulto recolectado, conservado en alcohol. El especímen fue hallado debajo de grandes rocas en un espacio abierto con predominancia de "pasto dulce" (Paspalum notatum) en el Parque Nicolás Avellaneda, en el barrio homónimo, Ciudad Autónoma de Buenos Aires, Argentina.

Comentarios. - Se trata de una especie fácilmente identificable; es una babosa de relativamente gran tamaño (lonfitud máxima: $6 \mathrm{~cm}$ ), color gris oscuro con quilla media dorsal desde el escudete al extremo caudal. El manto o escudo tiene forma de herradura y el neumostoma está ubicado en porción media distal del manto , la limacela (resto de conchilla) es ovalada y brillante. Anatomía interna: aparato genital distal con espermateca oval y ducto de la bursa copulatrix robusto, vagina muy corta, ducto o vaso deferente finaliza en el ápice del epifalo, y pene de forma irregular, casi del tamaño de la mitad del epifalo (Virgillito, 2012).

M. gagates parece ser originaria de las Islas Canarias y zonas costeras del Mar Mediterráneo (Barker, 1999). Es una especie invasora y se ha distribuido en gran parte de Europa, Japón, América del Norte, América del Sur, Bermudas, Australia, Nueva Zelanda y África del Sur (Barker, 1999; Campos y Calvo 2006), siempre asociada a ambientes disturbados y periurbanos (Clemente et al., 2010).

En Argentina, la especie cuenta con citas previas para la Isla Martín García, Neuquén y Río Negro (Fernández, 1973; Virgillito, 2012). En el sureste de la provincia de Buenos Aires, Milax gagates causa reducciones en los rendimientos de soja, girasol y oleaginosas al dañar las semillas y plántulas (Clemente et al., 2007, 2010).

El presente registro constituye el primero de la especie para la Ciudad de Buenos Aires.

Arthropoda Latreille, 1829

Diplopoda de Blainville, 1844

Julidae Leach, 1814

Cylindroiulus latestriatus (Curtis, 1845)

Material referido. - CFA-Ar-277, 3 ejemplares adultos recolectados, conservados en alcohol. Los especímenes fueron hallados debajo de troncos en una arboleda exótica de Álamo blanco (Populus alba) en el Parque Centenario, barrio de Caballito, Ciudad Autónoma de Buenos Aires, Argentina. 

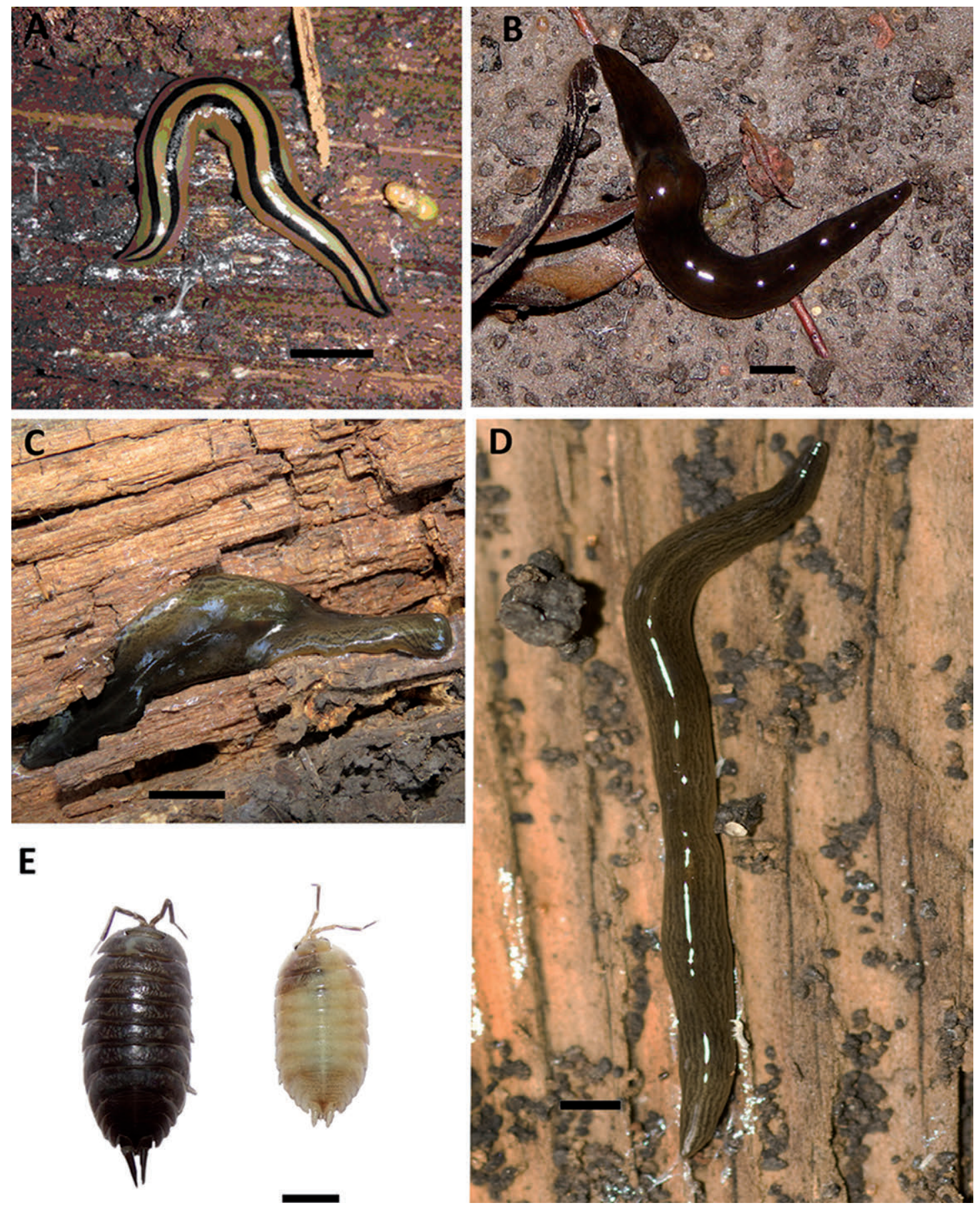

Figura 1. A, Imbira flavonigra (Tricladida), ejemplar adulto observado y recolectado (CFA-In-5602) en el Jardín Botánico "Carlos Thays", Ciudad Autónoma de Buenos Aires; B-D, Obama nungara (Tricladida), ejemplares adultos no recolectados B, D, Ramos Mejía, Buenos Aires, Argentina; C, Moreno, La Reja, Buenos Aires, Argentina; E, Cylisticus convexus (CFA-Ar-280) (Isopoda) a la izquierda se observa un ejemplar con coloración normal, a la derecha un ejemplar de coloración anómala. Escala: $5 \mathrm{~mm}$.

Figure 1. A, Imbira flavonigra (Tricladida), adult specimen observed and collected (CFA-In-5602) in the "Carlos Thays" Botanical Garden, Buenos Aires City; B-D, Obama nungara (Tricladida), adult specimens not collected B, D, Ramos Mejía, Buenos Aires, Argentina; C, Moreno, La Reja, Buenos Aires, Argentina; E, Cylisticus convexus (CFA-Ar-280) (Isopoda) on the left, a specimen with normal coloration is observed, on the right, a specimen with abnormal coloration. Scale: $5 \mathrm{~mm}$. 
Comentarios. - Cylindroiulus latestriatus es una especie de tamaño mediano (aproximadamente $3 \mathrm{~cm}$ de longitud) que puede distinguirse de taxones cercanos, por su cuerpo color pardo, con ozadenes muy oscuros, lo que resulta en una coloración general negruzca (Blower, 1985).

La especie, de origen posiblemente europeo se distribuye en el noreste de Europa, Canadá, Estados Unidos, Antártida, México y Chile (Blower, 1985; Shelley et al., 2014).

El presente constituye el primer registro de la especie para Argentina.

Ophyiulus pilosus (Newport, 1843)

Material referido. - CFA-Ar-278, 2 ejemplares adultos recolectados, conservados en alcohol. Fueron hallados debajo de grandes rocas en un espacio abierto con predominancia de "pasto dulce" (Paspalum notatum) en el Parque Nicolás Avellaneda, en el barrio homónimo, Ciudad Autónoma de Buenos Aires, Argentina.

Comentarios. - O. pilosus es una especie pequeña y delgada, de color negruzco, oscuro, la superficie de su cuerpo se encuentra cubierta por numerosas cerdas, en especial en la región media y posterior del animal, su extremo caudal es relativamente elongado y culmina en una espina anal traslúcida (Rolfe, 1934).

La especie, posiblemente originaria de América del Norte se distribuye a lo largo de Europa, Estados Unidos, Canadá, Nueva Zelanda y Tasmania (Dawson, 1958).

El presente constituye el primer registro de la especie para Argentina.

Paradoxomatidae Daday, 1889

Brachydesmus superus Latzel, 1884

Material referido. - CFA-Ar-279, 1 ejemplar adulto recolectado, conservado en alcohol. El especímen fue hallado debajo de ladrillos en un jardín interno en el barrio de Liniers, Ciudad Autónoma de Buenos Aires, Argentina.

Comentarios. - Brachydesmus superus se distingue fácilmente de otros taxones cercanos por su tamaño pequeño (no supera los $10 \mathrm{~mm}$ de longitud), cuerpo con 19 segmentos con las carenas laterales nítidamente incisas y su coloración general amarillo pálida, con una línea media pardo oscura (Schubart, 1954).

Es una especie ampliamente distribuida en Europa, Rusia, Islas Canarias y Estados Unidos (Schubart, 1954). Ha sido citada previamente para Saavedra y la Ciudad de La Plata (Schubart, 1954). Mauriès (1998) lo lista como presente en la provincia de Buenos Aires.

Este constituye el primer registro de la especie para la ciudad de Buenos Aires y el segundo para el país. 
Symphyla Ryder, 1880

Scolopendrellidae Bagnall, 1913

Symphylella vulgaris Hansen, 1903

Material referido. - CFA-Ar-280, 3 ejemplares adultos recolectados, conservados en alcohol. Los individuos fueron hallados debajo de ladrillos en un jardín interno en el barrio de Liniers, Ciudad Autónoma de Buenos Aires, Argentina. A semejanza de otros Scolopendrellidae, los individuos observados son distintivos por su cuerpo delgado y movimientos lentos, un rasgo compartido con otros Scolopendrellidae (véase Scheller, 1998).

Comentarios. - Los ejemplares son incluidos en el género Symphylella por presentar el primer par de patas reducido, vestigial (Scheller, 1998; Scheller y Adis, 1996). Dentro del género es referible a $S$. vulgaris por presentar una longitud corporal entre 1.9-2.4 milímetros, styli con una espina apical en su extremo terminal y margen posterior de los tergitos recto (Domínguez-Camacho y Vandenspiegel, 2012; Tabacaru et al., 2017).

La especie $S$. vulgaris es muy común y ampliamente distribuida en los jardines de casi todo el mundo, siendo especialmente peridoméstica y muy abundante en ambientes urbanos (Scheller y Stoev, 2006). Originaria de Europa, se la conoce en casi todos los países de ese continente, así como en Asia, África, Estados Unidos, México, y Nueva Zelanda (Scheller y Stoev, 2006).

Los Symphyla de Argentina se encuentran muy mal conocidos, en particular la familia Scolopendrellidae, por lo tanto, cualquier registro constituye una adición de vital importancia (Scheller, 1998). El presente hallazgo constituye el primero para la especie en Argentina.

\author{
Crustacea Brünnich, 1772 \\ Isopoda Latreille, 1817 \\ Oniscidea Latrielle, 1802 \\ Cylisticidae Verhoeff, 1949 \\ Cylisticus convexus (De Geer, 1778)
}

Material referido. - CFA-Ar-283, 8 ejemplares adultos recolectados, conservados en alcohol. Fueron hallados debajo de un gran tronco de plátano (Platanus $x$ hispanica) en un espacio abierto con predominancia de "pasto dulce" (Paspalum notatum) rodeado de árboles exóticos en el Parque Nicolás Avellaneda, en el barrio homónimo, Ciudad Autónoma de Buenos Aires, Argentina. Los ejemplares recolectados fueron observados junto a otros 50 individuos. Entre los ejemplares recolectados, dos de ellos carecen de pigmentación (Figura 1E).

Comentarios. - Cylisticus convexus se caracteriza por una combinación única de rasgos: cuerpo grande y ancho (hasta $12,5 \mathrm{~mm}$. de longitud total y $5.5 \mathrm{~mm}$, de ancho máximo), muy convexo (más que en Porcellio, pero menos que en Armadillidium) y 
con capacidad de arrollarse sobre sí mismo, flagelo de las antenas compuesto por dos segmentos subiguales, segmento caudal del cuerpo terminado en punta y coloración dorsal del cuerpo pardo grisácea (Giambiagi, 1935; véase Schmidt, 2003).

Se trata de una especie ampliamente distribuida en Europa y América del Norte (Schmidt, 2003). El único hallazgo previo registrado para la especie en Argentina corresponde a Giambiagi (1935) quien la menciona para Vélez Sarsfield, en la Ciudad de Buenos Aires.

La presente constituye la cita de la especie para Argentina luego de casi 90 años sin registros.

Arachnida Lamarck, 1801

Uloboridae Thorell, 1869

Uloborus plumipes Lucas, 1846

Material referido. - CFA-Ar-281, 1 ejemplar macho adulto recolectado, conservado en alcohol. El individuo fue hallado en su tela entre plantas en un jardín interno en el barrio de Liniers, Ciudad Autónoma de Buenos Aires, Argentina. La especie es muy abundante en dicha localidad, donde hay individuos cuya coloración varía del pardo negruzco al amarillento, tal como fuera remarcado por autores previos (Hänggi y Straub, 2016). Se contabilizaron más de 20 individuos.

Comentarios. - El ejemplar recolectado puede ser referible a Uloborus plumipes por presentar la siguiente combinación única de caracteres: la fila de ojos posteriores fuertemente recurvada, de manera que los bordes posteriores de los ojos medios se ven por delante de los mismos, cefalotórax piriforme, con el área cefálica conspicuamente angostada, dos grandes bultos en el abdomen y largos y densos pelos cubriendo la tibia del primer par de patas (Grismado, 2008; Tanikawa, 2017).

Los ejemplares observados se encontraban reposando en sus telas orbiculares de orientación sub-horizontal, colgando boca abajo y con las patas delanteras estiradas hacia delante, un comportamiento típico de esta especie (Suvàk, 2013).

Uloborus plumipes es originaria de África oeste y central (Rozwalka et al., 2013), y ha sido registrada para Asia, Europa y Sudamérica (Argentina) (Plattnick, 2013).

Se trata de una especie peridoméstica, muy frecuente en plantaciones de viveros (Reiche y Schmidt, 1994). Con el transporte antrópico de dichas plantas ornamentales la especie puede dispersarse rápidamente (Rozwalka et al., 2013). U. plumipes constituye el ejemplo de una especie invasora que desde contar con apenas un solo registro local puede convertirse en abundante en todos lados en menos de una década, tal como ha sido mencionado para Polonia (Rozwalka et al., 2013). En este sentido, Hänggi y Straub (2016) la incluyen entre las cinco especies invasoras de araña más común en las ciudades de Suiza.

En Argentina la especie cuenta apenas con una mención (sin cita de ejemplares concretos) en la ciudad de Buenos Aires (Grismado, 2008). La cantidad de individuos observados, incluyendo machos y hembras, indica una población reproductiva, en buen estado de salud y permanente. 
Tetragnathidae Menge, 1866

Leucauge argyra (Walckenaer, 1842)

Material referido. - CFA-Ar-282, 1 ejemplar hembra adulto recolectado, conservado en alcohol. El individuo fue hallado en su tela entre plantas en un jardín interno en el barrio de Liniers, Ciudad Autónoma de Buenos Aires, Argentina.

Comentarios. - Leucauge argyra es una especie que se diferencia de las formas cercanas sobre la base de su patrón de coloración, incluyendo tres líneas negras semi paralelas que a mitad de la región abdominal se curvan internamente para luego continuar semi paralelas hasta el extremo distal del abdomen (Walckenaer, 1842; Levi, 1980).

L. argyra se distribuye desde Estados Unidos hasta Brasil (Levi, 1980), siendo muy frecuente en zonas disturbadas por el accionar del hombre, especialmente cultivos (Cruz Pérez et al., 2015). Se la ha registrado como peridoméstica en áreas metropolitanas de Brasil (Silva Melo et al., 2014), México (Durán Barrón et al., 2009) y Cuba (Armas, 2003).

En Argentina cuenta con una primera y única cita publicada en la provincia de Corrientes, en monocultivos de bosques de pino y eucalipto (Avalos et al., 2009, 2018).

\section{DISCUSIÓN}

En Europa se ha constatado un rápido incremento en el número de especies introducidas de invertebrados, debido posiblemente a un incremento en el transporte e intercambio de bienes tanto dentro de Europa como hacia otros continentes (Rozwalka et al., 2013). Este patrón puede verse también reflejado en Argentina, donde nuevas especies de invertebrados invasores han sido mencionadas recientemente (Agnolin et al., 2019). Los nuevos hallazgos de invertebrados que corresponden a la presente contribución constituyen elementos novedosos que permiten bosquejar algunas hipótesis con respecto a la distribución y presencia de especies invasoras en ambientes urbanos en Argentina.

En Argentina los registros de planarias terrestres, miriápodos diplópodos y sínfilos exóticos son escasos, a pesar de ser formas relativamente abundantes en la mayor parte de las recolecciones y relevamientos faunísticos. Lamentablemente, el desconocimiento acerca de la distribución e incluso presencia en el país de especies exóticas de varios grupos de invertebrados en ambientes urbanos es alto, por lo tanto aún se desconoce el número aproximado de especies exóticas que podrían haber ingresado. De esta manera, se ignora si las especies exóticas se encuentran en expansión geográfica o demográfica. Específicamente en el caso de los miriápodos, las últimas revisiones sobre sínfilos y diplópodos argentinos (Mauriés, 1998; Scheller, 1998) coinciden en señalar que aún no se conoce prácticamente nada sobre las especies invasoras del grupo, careciéndose de información concreta de referencia y localidades precisas (ver Blower, 1985; Mauriés, 1998). Del mismo modo, a pesar 
que el conocimiento de los moluscos invasores en Argentina ha recibido atención en los últimos años (véase por ejemplo Virgillito, 2012; Virgillito y Miquel, 2013; Gutiérrez-Gregoric et al., 2013), aún se carece de datos de abundancia, dispersión, diversidad, taxonomía, entre otros aspectos. Debido a todas estas dificultades, aún no es posible establecer patrones de dispersión, demografía y diversidad de miriápodos, moluscos y planarias invasores.

A nivel mundial se han publicado pocos estudios sobre las características ecológicas de los conjuntos de isópodos urbanos (estructura de la comunidad, diversidad y relaciones de abundancia; Korsòs et al., 2002). En los paisajes urbanos de América del Norte las especies exóticas son a menudo muy abundantes y, por lo tanto, potencialmente podrían jugar un papel importante en el procesamiento de detritus (Hornung et al., 2015). La preferencia de estos isópodos introducidos (Cylisticus convexus, Porcellio laevis, Armadillidium vulgare) por alimentarse con semillas de plantas nativas sugiere que podrían representar una amenaza considerable para los ecosistemas autóctonos (Singer et al., 2012). Sin embargo, aún se carecen de estudios cuantitativos que permitan caracterizar el papel ecológico de estos invasores en ambientes urbanos, en especial en lo que a América del Sur se refiere.

Con respecto a los arácnidos, aún se carece de contribuciones que analicen en detalle las comunidades sinantrópicas. Hänggi y Straub (2016) hacen incapié sobre esta falta de información, que parece repetirse a lo largo del globo. Hänggi (2003) indica que existen numerosas especies de arañas invasoras en Europa, muchas de ellas en jardines y habitaciones privadas e incluso entre los cargamentos de frutas y verduras que abastecen los mercados. Algunas de esas especies representan hallazgos singulares y otras parecen haber establecido poblaciones perdurables en el tiempo.

Hänggi y Straub (2016) indican que sus investigaciones evidencian que las interpretaciones sobre la dispersión e invasión de especies necesitan de un buen conocimiento e información publicada, lo cual no es el caso de los arácnidos sinantrópicos. Dichos autores, sugieren que los viveros y tiendas de venta de plantas ornamentales, así como los jardines botánicos podrían actuar como peldaños que permitirín a las especies invasoras establecerse y dispersarse a lo largo de los ambientes urbanos. Esto es el resultado del intercambio y venta de plantas extranjeras, incluyendo el traslado de la tierra de las macetas entre jardines. Esta hipótesis también podría aplicarse a Argentina, aunque la evidencia aún es débil. Hänggi y Straub (2016) hacen hincapié sobre la virtual falta de información insistiendo que se necesitan con urgencia estudios sobre fauna urbana, incluso sobre especies que se consideran "comunes".

Rozwalka et al. (2013) argumentan para el caso de los arácnidos invasores que el efecto sobre la aracnofauna local podría ser poco importante. Debido a requerimientos específicos de temperatura y humedad, la distribución de especies invasoras se restringe a jardines privados y locaciones semejantes. En estos ambientes solo parecerían interferir con viejas especies sinantrópicas, por lo que no serían de peligro para la aracnofauna nativa.

La presente contribución confirma la constante invasión de especies exóticas en la Ciudad de Buenos Aires. En la actualidad hay un gran desconocimiento de varias especies introducidas de invertebrados, incluso de aquellas potencialmente perjudiciales para los ambientes nativos. Como remarcan Hänggi y Straub (2016), 
cualquier información de alguna de dichas especies importadas debería ser formalmente publicada y ser conocida por la comunidad científica.

\section{AGRADECIMIENTOS}

Agradecemos especialmente a C. Schmidt, quien puso a disposición bibliografía sobre isópodos terrestres. Especial agradecimiento a Sergio Bogan (FHN) por su ayuda durante la revisión de las colecciones a su cargo. Asimismo, agradecemos los comentarios y críticas sobre malacofauna terrestre en general que hemos recibido de S. Miquel (Museo Argentino de Ciencias Naturales "Bernardino Rivadavia"), así como la disección sobre el ejemplar aquí analizado de Milax gagates. Finalmente, se agradece a los revisores anónimos, cuyas observaciones han mejorado en gran medida la calidad del presente manuscrito.

\section{FINANCIAMIENTO}

No se contó con financiamiento para realizar este estudio.

\section{PARTICIPACIÓN}

Todos los autores recolectaron los especímenes de Ciudad Autónoma de Buenos Aires. Todos los autores participaron de la construcción del manuscrito.

\section{CONFLICTOS DE INTERÉS}

No se declaran conflictos de intereses con terceros.

\section{LITERATURA CITADA / BIBLIOGRAPHY}

Agnolin, F., Agnolin, A., Guerrero, E. (2019). Invertebrados exóticos nuevos o poco conocidos en la ciudad de Buenos Aires. Acta Zoológica Lilloana, 62, 48-67.

Álvarez-Presas, M., Mateos, E., Tudó, À., Jones, H., Riutort, M. (2014). Diversity of introduced terrestrial flatworms in the Iberian Peninsula: a cautionary tale. PeerJ, 2, e430.

Amaral, S.V., Ribeiro, G.G., Müller, M.J., Valiati, V.H., Leal-Zanchet, A. (2018). Tracking the diversity of the flatworm genus Imbira (Platyhelminthes) in the Atlantic Forest. Organisms, Diversity and Evolution, 18, 87-99.

Armas, L.F. (2003). Notas sobre los arácnidos de mi casa (Chelicerata, Arachnida). Revista Ibérica de Aracnología, 8, 143-149. 
Avalos, G., Damborsky, M.P., Bar, M.E., Oscherov, E.B., Porcel, E.A. (2009). Composición de la fauna de Araneae (Arachnida) de la Reserva Provincial Iberá, Corrientes, Argentina. Revista de Biología Tropical, 57, 339-351.

Avalos, G., Achitte-Schmutzler, H.C., De los Santos, M.E. (2018). Caracterización de la fauna de arañas en monocultivos de Eucalyptus y Pinus de la Reserva del Iberá, Corrientes, Argentina. Revista Mexicana de Biodiversidad, 89, 134-148.

Barker, G.M. (1999). Naturalised terrestrial Stylommatophora (Mollusca: Gastropoda). Fauna of New Zealand, 38, 1-247.

Blower, J. G. (1985). Millipedes. Synopses of the British Fauna (New Series) 35. London: Linnean Society.

Campos, J., Calvo, A. (2006). Moluscos introducidos en Uruguay. Comunicaciones de la Sociedad Malacológica del Uruguay, 9 (89), 75-78.

Carbayo, F., Álvarez-Presas, M., Jones, H., Riutort, M. (2016). The true identity of Obama (Platyhelminthes: Geoplanidae) flatworm spreading across Europe. Zoological Journal of the Linnean Society, 177, 5-2.

Clemente, N.L., Faberi, A.J., Salvio, C., López, A.N., Manetti, P.L., Monterubbianesi, M.G., Álvarez Castillo, H.A. (2007). Estimación de los daños de babosas (Mollusca: Pulmonata) en el cultivo de girasol en siembra directa. En Sistema Nacional Argentino de Vigilancia y Monitoreo de Plagas, IV Congreso Argentino de Girasol (397-398). Buenos Aires: Argentina.

Clemente, N.L., Faberi, A.J., Salvio, C., López, A.N. (2010). Biology and individual growth of Milax gagates (Draparnaud, 1801) (Pulmonata: Stylommatophora). Invertebrate Reproduction \& Development, 54, 163-168.

Cruz-Pérez, A., Pérez-De la Cruz, M., Sánchez-Soto, S., Torres de la Cruz, M. (2015). Fluctuación poblacional de arañas (Araneae: Tetragnathidae, Pholcidae) en el agroecosistema cacao en Tabasco, México. Revista Colombiana de Entomología, 41, 132-138.

Dawson, E.W. (1958). Exotic Millipedes (Diplopoda) in New Zealand. N.Z. Entomologist, 2, 1-5.

Domínguez Camacho, M., Vandenspiegel, D. (2012). Scolopendrellidae (Myriapoda, Symphyla) from the afrotropics with descriptions of seven new species. European Journal of Taxonomy, 32, 1-28.

Durán-Barrón, C.G., Francke, O.F., Pérez-Ortiz, T.M. (2009). Diversidad de arañas (Arachnida: Araneae) asociadas con viviendas de la ciudad de México (Zona Metropolitana). Revista Mexicana de Biodiversidad, 80, 55-69.

Fernández, D. (1973). Catálogo de la malacofauna terrestre argentina. La Plata: Comisión de Investigaciones Científicas.

Giambiagi, D. (1935). Isópodos nuevos para la fauna argentina. Physis, 11, 509.

Grismado, C.J. (2008). Uloboridae. In: Claps, L.E., Debandi, G., Roig, S. (eds). Sociedad Entomológica Argentina, Biodiversidad de Artrópodos Argentinos, 97103.

Gutiérrez Gregoric, D.E., Beltramino, A.A., Vogler, R.E., Cuezzo, M.G., Núñez, V., Gomes, S.R., Virgillito, M., Miquel, S.E. (2013). First records of four exotic slugs in Argentina. American Malacological Bulletin, 31, 245-256. 
Hänggi, A. (2003). Nachträge zum «Katalog der schweizerischen Spinnen». Neunachweise von 1999 bis 2002 und Nachweise synanthroper Spinnen. Arachnologische Mitteilungen, 26, 36-49.

Hänggi, A., Straub, S. (2016). Storage buildings and greenhouses as stepping stones for non-native, potentially invasive spiders (Araneae) - a baseline study in Basel, Switzerland. Arachnologische Mitteilungen, 51, 1-8.

Hornung, E., Szlavecz, K., Dombos, M. (2015). Demography of some non-native isopods (Crustacea, Isopoda, Oniscidea) in a Mid-Atlantic forest, USA. ZooKeys, $515,127-143$.

Hyman, L.H. (1943). Endemic and exotic land planarians in the United States with a discussion of necessary changes of names in the Rhynchodemidae. American Museum Novitates, 1241, 1-21.

Jones, H.D. (1998). The African and European land planarian faunas, with an identification guide for field workers in Europe. Pedobiologia, 42, 477-489.

Justine, J., Winsor, L., Gey, D., Gros, P., Thévenot, J. (2020). Obama chez moi! The invasion of metropolitan France by the land planarian Obama nungara (Platyhelminthes, Geoplanidae) PeerJ, 8, e8385.

Korsós, Z., Hornung, E., Szlavecz, K., Kontschán, J. (2002). Isopoda and Diplopoda of urban habitats: new data to the fauna of Budapest. Annales historico-naturales Musei nationalis hungarici, 94, 193-208.

Lago-Barcia, D., Fernández-Álvarez, F.A., Negrete, L., Brusa, F., Damborenea, C., Grande, C., Noreña, C. (2015). Morphology and DNA barcodes reveal the presence of the non-native land planarian Obama marmorata (Platyhelminthes: Geoplanidae) in Europe. Invertebrate Systematics, 29 (1), 12.

Lago-Barcia, D., Fernández-Álvarez, F.A., Brusa, F., Rojo, I., Damborenea, C., Negrete, L., Grande, C., Noreña, C. (2019). Reconstructing routes of invasion of Obama nungara (Platyhelminthes: Tricladida) in the Iberian Peninsula. Biological Invasions, 21, 289-302.

Levi, H.W. (1980). The orbweaver genus Mecynogea, the subfamily Metinae andthe genera Pachygnatha, Glenognatha and Azilia of the subfamily Tetragnathinae North of Mexico (Araneae: Araneidae). Bulletin of the Museum of Comparative Zoology, 149, 1-74.

Mauriés, J. P. (1998). Diplopoda. En Biodiversidad de Artrópodos Argentinos, una perspectiva biotaxonómica. Buenos Aires: Ediciones Sur.

Negrete, L.H. (2013). Diversidad de planarias terrestres (Platyhelminthes, Tricladida, Geoplanidae) en el bosque paranaense argentino. La Plata, Buenos Aires: Universidad Nacional de la Plata-Facultad de Ciencias Naturales y Museo.

Platnick, N.I. (2013). The world spider catalog, versión 13.5. American Museum of Natural History. Disponible enhttp://research.amnh.org/iz/spiders/catalog

Reiche, W., Schmidt, G. (1994). Weitere Nachweise von Uloborus plumipes in Deutschland. Arachnologische Mitteilungen, 7, 50-51.

Rolfe, S.W. (1934). Notes on Diplopoda I. The re-study of a widely distributed British millipede, Ophyiulus pilosus (Newport). Annals \& magazine of natural history, 14 (80), 192-203. 
Rozwałka, R., Rutkowski, T., Bielak-Bielecki, P. (2013). New data on introduced and rare synanthropic spiders (Arachnida: Araneae) in Poland. Annales Universitatis Mariae Curie-Sk³ odowska, 68, 127-150.

Scheller, U. (1998). Pauropoda (Myriapoda) from Portugal. Stobaeana, 11, 1-7.

Scheller, U., Adis, J. (1996). A pictorial key for the symphylan families and genera of the Neotropical region south of central Mexico (Myriapoda, Symphyla). Studies on Neotropical Fauna and Environment, 31, 57-61.

Scheller, U., Stoev, P. (2006). First records of Symphyla (Myriapoda) from Bulgaria. Historia naturalis bulgarica, 17, 35-37.

Schmidt, C. (2003). Contribution to the phylogenetic system of the Crinocheta (Crustacea, Isopoda). Part 2. (Oniscoidea to Armadillidiidae). Mitteilungen aus dem Museum fûr Naturkunde in Berlin. Zoologische Reihe, 79, 3-180.

Schubart, O. (1954). Diplopodos Argentinos del Museo de la Ciudad Eva Perón. III. Fam. Stylodesmidae, IV. Fam. Cryptodesmidae, V. Fam. Polydesmidae. Ministerio de educación de la nacion. Universidad nacional de Eva Perón. Notas del Museo, 17, 347-358.

Shelley, R.M., Morrill, E.D., Faber, D.A. (2014). A julid milliped in Chilean Patagonia, and a compilation of ordinal representatives in South America and associated islands (Diplopoda: Julida). Insecta Mundi, 875, 1-8.

Silva Melo, T., Lima Peres, M.C., Chavari, J.L., Domingos, A., Brescovit, J.H., Delabie, C. (2014). Ants (Formicidae) and Spiders (Araneae) listed from the Metropolitan Region of Salvador, Brazil. Check List, 10, 355-365.

Singer, C., Bello, N.M., Snyder, B.A. (2012). Characterizing prevalence and ecological impact of non-native terrestrial isopods (Isopoda, Oniscidea) in tallgrass prairie. Crustacean, 85, 1499-1511.

Soors, J., Van den Neucker, T., Halfmaerten, D., Neyrinck, S., De Baere M. (2019). On the presence of the invasive planarian Obama nungara (Carbayo, ÁlvarezPresas, Jones \& Riutort, 2016) (Platyhelminthes: Geoplanidae) in an urban area in Belgium. Belgian Journal of Zoology, 149 (1), 43-47.

Suvák, M. (2013). Invasive spider Uloborus plumipes Lucas, 1846 (Araneae: Uloboridae), new to Slovakia. Folia Faunistica Slovaca, 18, 39-45.

Tabacaru, I.G., Giurginca, A. Cãtãlin Baba, S. (2017). Identification key to the Symphyla of Romania. Travaux de LInstitute de Speologie Emil Racovita, 6, 3-23.

Tanikawa, A. (2017). Uloborus plumipes and Dipoena pelorosa (Araneae: Uloboridae, Theridiidae): two newly recorded spiders in Japan. Acta Arachnologica, 66(1), 5-8.

Vila-Farré, M., Sluys, R., Mateos, E., Romero, R. (2011). Land planarians (Platyhelminthes: Tricladida: Geoplanidae) from the Iberian Peninsula: new records and description of two new species, with a discussion on ecology. Journal of Natural History, 45, 869-891.

Virgillito, M. (2012). Panorama de los gastrópodos terrestres exóticos en Argentina (Gastropoda Pulmonata Stylommatophora). Facultad de Ciencias Exactas y Naturales, Universidad de Buenos Aires, Buenos Aires. Tesis de grado, inédita. 
Virgillito, M., Miquel, S.E. (2013). New records of exotic land snails and slugs in Argentina. Revista del Museo Argentino de Ciencias Naturales Nueva Serie, $15,295-303$.

Walckenaer, C.A. (1842). Histoire naturelle des Insects. Aptères. Paris: Librairie Encyclopédique de Roret. 